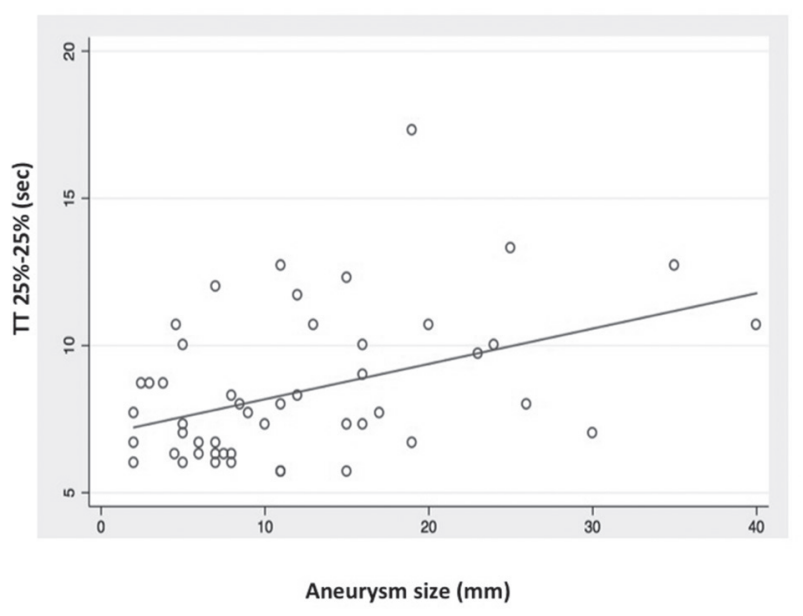

Abstract 0-041 Figure 1

Introduction Treatment of large and giant intracranial aneurysms are often associated with larger complications rates, related to hemodynamic changes of intracranial flow distal to the intracranial aneurysms. In this paper we evaluated the baseline contrast transit times on angiography for patients with proximal anterior circulation aneurysms.

Methods Medical charts and digital subtraction angiographic (DSA) films for patients with intracranial aneurysms were reviewed. Only proximal, unruptured anterior circulations aneurysms were included. DSA images were analyzed over M1 segment, using custom made software for the time-density. Analysis included $\mathrm{TT}_{10 \%-100 \%}$ (time needed for the contrast to change from 10\%-100\% image intensity), $\mathrm{TT}_{100 \%-10 \%}$ (time needed for the contrast to change from 100\%-10\% image intensity), and $\mathrm{TT}_{25 \%-25 \%}$ (time needed for the contrast to change from $25 \%-25 \%$ image intensity. This was compared to the contralateral M1 transit times.

Results A total of 50 patients were included in this study. Aneurysm size ranged from $2-40 \mathrm{~mm}$, mean $12 \mathrm{~mm}$. Analysis over the M1 segment showed a significant increase in the $\mathrm{TT}_{25 \%-25 \%}$ (7.6 to 8.46 seconds, $P=0.006$ ) compared to the contralateral side. There was significant correlation (Spearman's correlation) between the $\mathrm{TT}_{10 \%-100 \%}$, the $\mathrm{TT}_{25 \%-25 \%}$ and the aneurysm size (rho $=0.291,0.362$ and $P=0.041$, 0.01 respectively) (Figure 1), indicating a slower contrast time

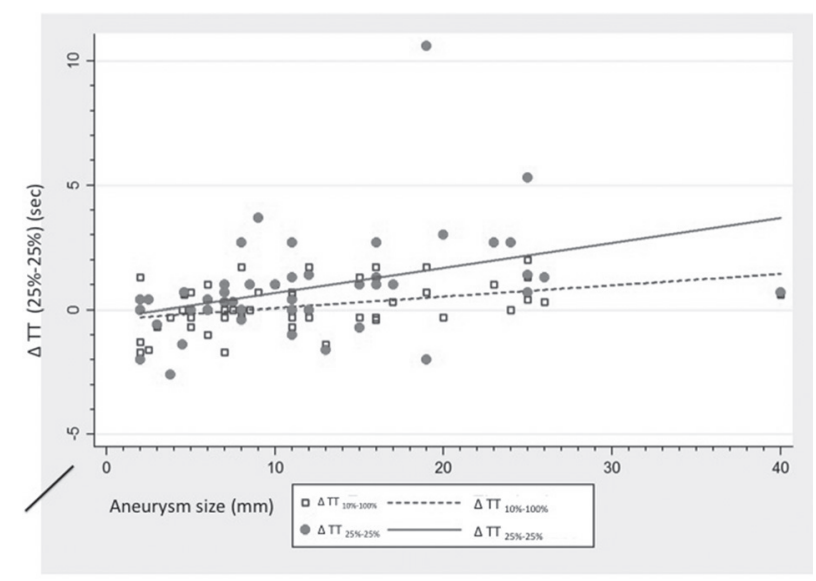

Abstract 0-041 Figure 2 in larger aneurysms. Similarly, there was significant correlation between the absolute difference between the ipsilateral and contralateral TTs and aneurysm size $\left(\mathrm{TT}_{10 \%-100 \%}: P=0.005\right.$, rho $=0.393 ; \mathrm{TT}_{25 \%-25 \%}: \mathrm{P}<0.001$, rho $=0.52$ ) (Figure 2).

Conclusion Our analysis shows that statistically significant difference in the intracranial contrast TT as a function of proximal aneurysm size. The mechanism for this might be related to the Windkessel effect where the aneurysm as a blood reservoir in systole and partially release the contrast in diastole. Changes to this baseline phenomenon might contribute to complications post flow diverters implementation.

Disclosures A. Hussein: None. A. Linninger: None. F. Charbel, II: None. C. Hsu: None. F. Charbel: 2; C; Transonic. 4; C; VasSol Inc. V. Aletich: 2; C; Codman. A. Alaraj: 2; C; Codman.

\section{0-042 FUNCTIONAL OUTCOME PREDICTION MODEL FOR CAROTID STENTING PATIENTS USING ADMISSION PROFILES: 29,453 PATIENTS USING NIS DATA 2005 TO 2013}

${ }^{1} \mathrm{~S}$ Park, ${ }^{2} \mathrm{M}$ Alexander, ${ }^{3} \mathrm{~A}$ Rosengart. ${ }^{1}$ Neuroscience, Albany Medical Center, Albany, NY; ${ }^{2}$ Neurosurgery, Cedars-Sinai Medical Center, Los Angeles, CA; ${ }^{3}$ Neurology, Cedars-Sinai Medical Center, Los Angeles, CA

\subsection{6/neurintsurg-2016-012589.42}

Introduction Carotid stenting (CAS) has been widely used since the seminal CREST trial, demonstrating equivalent results to carotid endarterectomy (CEA). With normal surgical risk patients having symptomatic or asymptomatic carotid artery disease, CAS and CEA, in qualified hands, have comparable outcomes, but patient-specific risk factors to estimate functional outcome were poorly studied. In practice, the choice between CEA and CAS is based on lesion anatomy and preference of patient and surgeon rather than considering defined risk factors associated with unfavorable outcome. We aimed to determine medical risk factors for poor functional outcome after CAS using the Healthcare Cost and Utilization Project Nationwide Inpatient Sample database (NIS) and formulate outcome prediction model to estimate unfavorable functional outcome.

Methods We included 29,453 patients (age $>\geq 18$ ) with symptomatic and asymptomatic carotid artery disease from 2005 to 2013, who underwent CAS using ICD-9 procedure code and diagnosis code for chronic and acute comorbidity as well as administration data including social economic and demographic profiles. Applying the non-parametric Jonckheere-Terpstra test, Multiple logistic regression, and multiple linear regression analyzes (SAS 9.4; SPSS 22) to formulate outcome prediction model using covariates for (1) Socio-economic status; age, sex and race, (2) chronic comorbidities; hypertension, diabetes mellitus, hyperlipidemia, coronary artery disease, congestive heart failure, atrial fibrillation/ flutter, COPD, chronic kidney disease, aortic/visceral/peripheral atherosclerosis, tobacco/alcohol dependence and morbid obesity, (3) care complexity; number of comorbidities, numbers of inpatient diagnosis/ procedures and (4) Acute comorbidities; acute myocardial infarct, acute kidney failure, pneumonia, acute respiratory failure and sepsis. We compared outcome markers which were defined as (1) procedure related stroke, (2) mortality, and (3) functional outcome based on discharge disposition; long term facility or not. Care complexity and acute comorbidities were also considered for outcome analysis without utilization for 
then outcome prediction formula because these variables are not predictable on admission.

Results Mean age of symptomatic and asymptomatic CAS patients was 70.3 years (SD \pm 10.6 years) with $20 \%>80$ years old; $39.5 \%$ females; $71.8 \%$ Whites, $4.5 \%$ Blacks and 4.1\% Hispanics. $16.2 \%$ had symptomatic carotid stenosis. As chronic comorbidities, $75.7 \%$ had HTN, 32.1\% DM, 58.1\% HLD, 54.3\% CAD, 0.6\% CHF, 10.4\% Atrial fibrillation/flutter $12.9 \%$ COPD, $11.4 \%$ chronic renal failure, $16.4 \%$ tobacco dependence, $0.4 \%$ alcohol dependence and 1.4\% morbid obesity. As outcome makers, in-patient mortality was 1.1\% (symptomatic $4.6 \%$ /asymptomatic $0.3 \%$ ), $2 \%$ of patient had procedure related stroke (symptomatic 9.6\%/asymptomatic $0.5 \%$ ), discharge disposition to long term facility 9.4\% (symptomatic $39.5 \%$ /asymptomatic $4.1 \%$ ). Multiple outcome models developed to predict defined outcome markers to select the best predictable model. Several variables identified Age (greater than 80$)$, presence of symptoms including TIA $(\mathrm{p}<0.001)$, CHF $(\mathrm{p}<0.001)$, COPD $(\mathrm{p}<0.001)$ and CRF $(\mathrm{p}<0.001)$ to formulate the best model. Long term facility disposition possibility $=0.012+0.067(1$, if age $>80,0$ if age $<80)$ $+0.014(1$, if $\mathrm{DM})+0.084(1$, if $\mathrm{CHF})+0.03(1$, if $\mathrm{CRF})$ $+0.030(1$, if COPD) +0.351 (1, if symptomatic) (Multiple linear regression, $\left.\mathrm{R}^{2} 0.205\right)$

Conclusions Age (greater than 80), presence of symptoms including TIA, DM, CHF, COPD and CRF were identified to predict worse functional outcome after CAS.

Disclosures S. Park: None. M. Alexander: 1; C; : Investigator in CREST2 Trial. A. Rosengart: None.

\section{Oral Poster Abstracts}

\section{P-001 PERMANENT IMPLANTATION OF THE SOLITAIRE DEVICE AS A BAILOUT TECHNIQUE FOR LARGE VESSEL INTRACRANIAL OCCLUSIONS}

1) Houde, ${ }^{2} \mathrm{E}$ Barber, ${ }^{3} \mathrm{M}$ Kelly, ${ }^{3} \mathrm{~L}$ Peeling. ${ }^{1}$ College of Medicine, University of Saskatchewan, Saskatoon, SK, Canada; ${ }^{2}$ General Surgery, University of Saskatchewan, Saskatoon, SK, Canada; ${ }^{3}$ Neurosurgery, University of Saskatchewan, Saskatoon, SK, Canada

\subsection{6/neurintsurg-2016-012589.43}

Introduction The Solitaire (Medtronic, Minneapolis, MN) is a stentriever device for endovascular treatment of acute ischemic stroke (AIS). The Solitaire is well described for use in temporary endovascular bypass, and mechanical thrombectomy. Permanent placement of the Solitaire is an option, but there are limited reports to date. The authors of this study review six cases of Solitaire stent implantation in the setting of large vessel occlusion and acute ischemic stroke, where temporary endovascular bypass and mechanical thrombectomy failed.

Materials and methods Six cases of failed mechanical thrombectomy are reviewed, including procedural review, timing of anti-platelet agents, and radiographic and clinical outcomes.

Results Six patients with severe, acute ischemic stroke (AIS) and large vessel occlusion (LVO) were triaged to mechanical thrombectomy. Despite multiple attempts at mechanical thrombus retrieval with a combination of devices including stentrievers and direct thrombus aspiration, flow could not be restored. As a bailout maneuver, the Solitaire stent was deployed and detached across the thrombus with TICI grade
3 flow restoration. All patients were loaded with full dose intra-arterial abciximab immediately after stent deployment and transitioned to dual antiplatelet therapy within 24 hours. There were no post-procedure hemorrhagic complications. All patients had significant neurological improvement post-procedurally, with favorable modified Rankin scores at follow-up.

Conclusion With the recent over-whelming evidence demonstrating the effectiveness endovascular therapy in acute ischemic stroke, the expansion of stroke therapy requires that a variety of techniques be available to the operators. Permanent implantation of the Solitaire stent achieves adequate distal flow and good clinical outcomes in the setting of AIS. This case series suggests this technique provides a safe alternative for difficult cases refractory to other revascularization techniques.

Key Words stroke, revascularization, stent

Disclosures J. Houde: None. E. Barber: None. M. Kelly: 2; C; Medtronic. 4; C; Blockade. 6; C; Penumbra. L. Peeling: None.

\section{P-002 INCIDENCE AND PROGNOSIS OF PLAQUE PROTRUSION DURING CAROTID ARTERY STENTING}

${ }^{1} \mathrm{~K}$ Takayama, ${ }^{1} \mathrm{~K}$ Myouchin, ${ }^{2} \mathrm{~T}$ Wada, ${ }^{3} \mathrm{M}$ Kotsugi, ${ }^{3} \mathrm{~S}$ Kurokawa, ${ }^{4} \mathrm{~K}$ Kichikawa. ${ }^{1}$ Radiology and Interventional Neuroradiology, Ishinnkai Yao General Hospital, Yao City, Japan; ${ }^{2}$ Radiology, Nara Medical University, Kashihara city, Japan; ${ }^{3}$ Neurosurgery, Ishinnkai Yao General Hospital, Yao City, Japan; ${ }^{4}$ Radiology, Nara Medical University, Kashihara, Japan

\subsection{6/neurintsurg-2016-012589.44}

Background Plaque protrusion (PP) is sometimes detected by angiography or intravascular ultrasound (IVUS) during carotid artery stenting (CAS). However, the incidence and prognosis of PP are unclear.. Purpose: We investigated the incidence and prognosis of PP during CAS.

Material and Methods We retrospectively analyzed the case of 292 patients with 308 carotid artery stenoses $(252 \mathrm{men}$; age: 39-97 years [mean: 73.4 years]; symptomatic stenosis: 137 lesions; mean stenosis rate: 78.4\%) who underwent CAS and preoperative MR plaque imaging from October 2007 to October 2015. The incidence of plaque protrusion was evaluated by IVUS and angiography. The 30 day prognosis of PP and the incidence of ischemic lesions within $48 \mathrm{~h}$ after CAS were assessed using diffusion-weighted images. All CAS was performed using the standard procedure and embolic protection devices.

Result PP occurred in eight patients (2.6\%). Stroke occurred in four patients (major: 1, minor: 3) (50.0\%) in the PP groups. New ischemic lesions were observed in $87.5 \%(7 / 8)$ of the PP group. There were siginificant difference in the frequencies of stroke and ischemic lesions between the PP group and non-PP group. The detection of unstable plaque on MR plaque imaging and the use of open cell stent were significant predictive factors of PP.

Conclusion PP during CAS is strongly associated with ischemic complications. PP prevention is necessary to avoid ischemic complications during CAS.

Disclosures K. Takayama: 2; C; Medtronic. K. Myouchin: None. T. Wada: None. M. Kotsugi: None. S. Kurokawa: None. K. Kichikawa: None. 